\title{
Soluciones de Bajo Costo Usando el Modelo Infraestructura como Servicio con Alta Disponibilidad y Virtualización
}

\section{(Low-Cost Solutions Using the Infrastructure as a Service with High Availability and Virtualization Model)}

\author{
Cesar Armando Moreira Zambrano¹, Walter Daniel Zambrano-Romero², Rene Guamán-Quinché3 \\ Wilner Geoberti Cuenca Álava ${ }^{4}$
}

\begin{abstract}
Resumen:
En esta publicación se presentan los resultados obtenidos de la implementación de una infraestructura para mejorar los servicios tecnológicos de correo electrónico, entorno virtual de aprendizaje, repositorio digital y biblioteca virtual en la Escuela Superior Politécnica Agropecuaria de Manabí, ESPAM, mediante la utilización de mecanismos de alta disponibilidad y virtualización para ofrecer recursos de mayor confiablidad. La virtualización es una tecnología potenciadora de vanguardia que está transformado el funcionamiento de servicios tecnológicos, pero, implica un cambio de paradigma en las tecnologías de la información orientada a los servicios y la computación en la nube. Para ejecutar cada uno de los procesos, se empleó, como estrategia, la metodología ciclo en V. Los servicios de virtualización fomentan que las empresas e instituciones transformen su forma de operar para estar a la vanguardia en innovación de sus servicios como solución tecnológica, es así que la implementación de tecnología redundante en la ESPAM, le ha permitido que sus servicios tecnológicos estén siempre operativos, en beneficio de la comunidad universitaria, ya que si se presentaren fallos en el sistema principal o los servicios, se habilitarán rápidamente los respaldos permitiendo que los sistemas entren en operación de inmediato.
\end{abstract}

Palabras Clave: Alta disponibilidad; Computación en la nube; Computación distribuida; Nube educativa; Virtualización.

\begin{abstract}
:
This paper presents the results obtained from the implementation of an infrastructure to improve technological services of email, virtual learning environment, digital repository and virtual library at the Polytechnic Agricultural Higher School of Manabí (Polytechnic School of Agriculture of Manabí), ESPAM, through the use of high availability and virtualization mechanisms to provide more reliable resources. Virtualization is an empowering and cutting-edge technology that is transforming the operation of technological services, but it involves a paradigm shift in serviceoriented information technologies and cloud computing. To execute each of the processes the V-cycle methodology was used as a strategy. Virtualization services empowers companies and institutions by transforming how they operate to be at the forefront of innovation in their services as a technological solution. So the implementation of redundant technology in the ESPAM, has allowed its technological services are always operative, for the benefit of the university community, because if there were failures in the main system or services, the backups will be enabled quickly allowing the systems come into operation immediately.
\end{abstract}

Keywords: High availability; Cloud computing; Distributed computing; Educational cloud; Virtualization.

\footnotetext{
1 Escuela Superior Politécnica Agropecuaria de Manabí, Calceta - Ecuador (cmoreira@espam.edu.ec)

1 Universidad Técnica de Manabí, Portoviejo - Ecuador (walter.zambrano@fci.edu.ec)

1 Universidad Nacional de Loja, Loja - Ecuador (rguaman@unl.edu.ec)

1 Universidad Técnica de Manabí, Portoviejo - Ecuador (wilner.cuenca@fci.edu.ec)
} 


\section{Introducción}

Las redes de Infraestructura como Servicio, también conocidas como laaS por su sigla del inglés Infrastructure as a Service, nos ofrecen la posibilidad de administrar de una manera más óptima los recursos de hardware; además, de analizar y evaluar el Software como Servicio, SaaS Software as a Service, y la Plataforma como Servicio, PaaS-Platform as a Service. La virtualización de los recursos de hardware posibilita contar con el hardware que se necesite, creando y destruyendo máquinas virtuales en tiempo real para adaptarse a la potencia requerida en todo momento, mejorando la calidad de estos servicios. De esta forma, para realizar la misma carga de trabajo, una red laaS necesitará menos recursos de hardware que una red de servidores convencionales.

La funcionabilidad de una laaS radica en una oferta altamente automatizada, donde los recursos informáticos, complementados con las capacidades de almacenamiento, se gestionan más eficientemente, por lo que se necesita que la Infraestructura como Servicio sea un modelo de provisión, en el que una organización externaliza el equipo utilizado para apoyar sus operaciones, incluido el almacenamiento, hardware, servidores y los componentes de red (Buyya, R. et al., 2009). En la actualidad, la informática persigue un modelo orientado a los servicios en tiempo real, a como se ofrecen los servicios de luz, agua, teléfono, gas, etc.

La informática para investigaciones, denominada computación de alto rendimiento, usa potentes herramientas y procesos de computación para generar datos en investigaciones académicas avanzadas. Con un clúster de computación de alto rendimiento, los centros de investigación pueden obtener la velocidad y potencia de una costosa supercomputadora a una fracción del costo y con menos riesgo de sufrir tiempos de inactividad prolongados (Chuquiguanca, L. et al., 2015)

En modelos existentes actuales, los servicios son otorgados en línea para los usuarios y están disponibles en el momento en que estos son requeridos. Además, los usuarios utilizan los servicios sin la preocupación de donde se encuentren, ejecutándolos desde sus equipos de cómputo. Así, los servicios de cómputo ofrecidos permiten realizar en línea todos los procesos de usuario y el almacenamiento de la información, en lugar de ejecutarlos en una computadora local. Esto conlleva, como consecuencia lógica, que el usuario requiera de equipos de cómputo menos sofisticados, con el consiguiente ahorro en la adquisición del equipo, por ende, disminución en el consumo de energía, así como menor gasto en software de aplicación.

El precio del hardware disminuye según los niveles de desarrollo de tecnología, haciendo posible a las empresas comprar servidores cada vez más potentes. Sin embargo, los gastos operacionales, como el mantenimiento de servidores, tienen un crecimiento progresivo, por lo que 
se hace necesario administrar mejor los recursos de hardware o incluso se puede pensar en externalizar este servicio (Zambrano, W. et al., 2015). La computación en la nube cuenta con recursos que se acceden desde cualquier sistema fijo o móvil, mediante el internet; los clientes utilizan únicamente los recursos que realmente necesitan, y pagan por el uso de ese servicio a un precio relativamente bajo en comparación con el costo de tener una infraestructura propia, misma que demanda elevados gastos operativos y financieros.

La reducción en el número de servidores implica una disminución de costos, tanto a nivel de adquisición de nuevos servidores como su mantenimiento, al reducirse las tareas de administración. Además, la reducción del número de servidores conlleva a una optimización del espacio físico, una disminución del consumo eléctrico, tanto en el consumo de los servidores como el de refrigeración. Por tanto, las soluciones de las laaS ofrecen a las empresas la posibilidad de un crecimiento más sostenible, tanto económico como medioambiental (Guirao Villalonga, A. 2011).

Además, el uso del clúster permite reducir el tiempo de resolución de problemas computacionales, más aun, resolver problemas que no cabrían en la memoria de un solo procesador secuencial. Para esto, es necesario utilizar sistemas de altas prestaciones y algoritmos paralelos que utilicen estos sistemas de manera eficiente (Sheharyar, A. \& Bouhali, O. 2014).

La investigación se centró en el estudio de la computación en la nube y la Infraestructura como Servicio y su viabilidad en la virtualización de los diferentes sistemas, optimizando recursos físicos y brindando soluciones de alta disponibilidad y bajo costo para la ESPAM.

\section{Metodología}

La investigación se realizó en la Escuela Superior Politécnica Agropecuaria de Manabí. Los materiales utilizados fueron: la Infraestructura como Servicio, el sistema de virtualización, sistemas de almacenamiento masivo y sistemas operativos tradicionales con la finalidad de proveer una alta disponibilidad y confiabilidad de operatividad de los servicios que ofrece la ESPAM. La ejecución se realizó mediante el método informático ciclo en $\mathrm{V}$, y se contemplaron las fases de: especificaciones, diseño de alto nivel y de detalle, implementación, y finalmente la del test unitario, de integración y operacional.

Las plataformas en nube de laaS son la tecnología innovadora, que garantiza la estabilidad de aplicaciones críticas, implementando el verdadero criterio de sistemas distribuidos que actualmente está alcanzando un nivel prometedor a las soluciones informáticas, garantizando seguridad y escalabilidad a través de mecanismos de redundancia. 


\subsection{Fase de Especificaciones}

En esta fase se detalla el análisis de los procedimientos que se realizan en el centro de datos de la ESPAM tomando como punto de partida las características del hardware que posee la institución, mediante una entrevista al personal que labora en el área de tecnología para verificar las necesidades y falencias de los equipos servidores, obteniéndose el detalle de los respectivos sistemas que posee la institución y su nivel de seguridad.

En los sistemas de computación en la nube se debe considerar que se puede contar con tres modelos de capas: SaaS, PaaS e laaS, cada uno puede ser usado en diferentes campos de la educación, a como se indica en la Figura 1 (Sociedad Española Informatica. 2011). En esta investigación se usó el modelo laaS como mecanismo de solución de bajo costo en la implementación de servidores virtualizados.

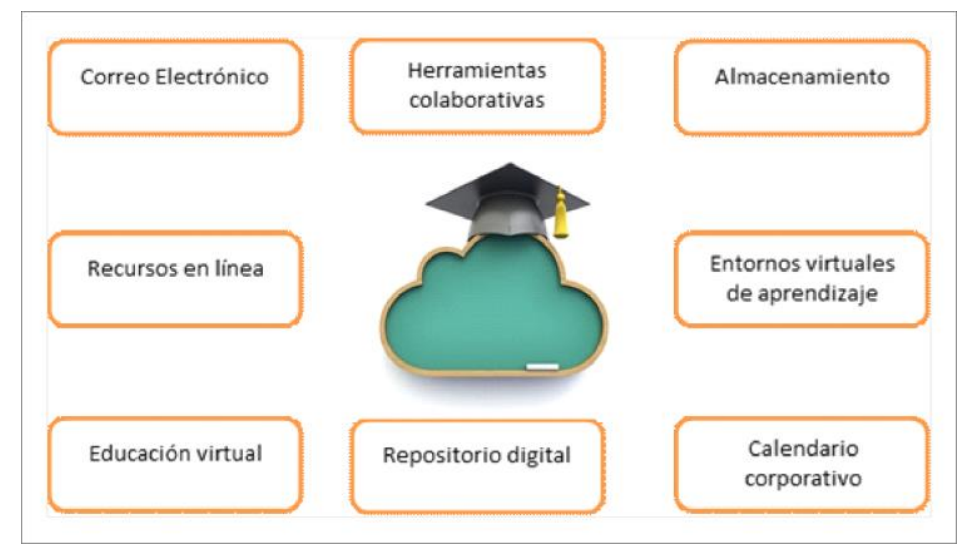

Figura 1. Modelos de computación en la nube en la educación.

\subsection{Beneficios del Uso de la Computación en la Nube en la Educación}

Los beneficios de contar con una infraestructura de laaS privada en un centro educativo, que imparta formación relacionada con las TIC, ofrece al alumnado la oportunidad de instalar, configurar y administrar diferentes plataformas de sistemas operativos, acrecentando su formación en un campo que, sin duda, es uno de los más compactos con un futuro prometedor en la informática actual (Nazareno, G. 2012). Figura 2. 


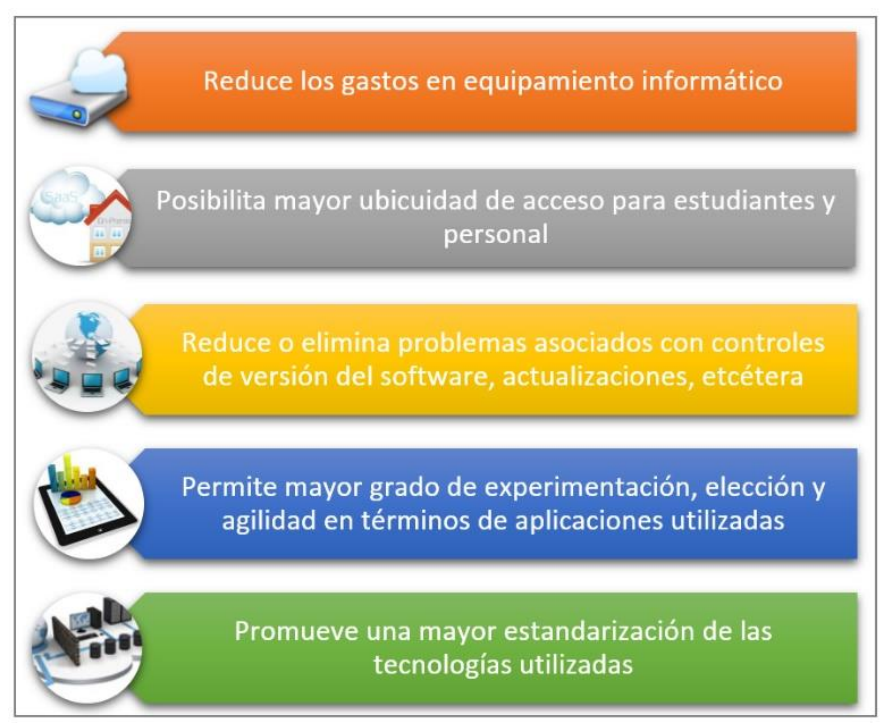

Figura 2. Beneficios del uso la computación en la nube en la educación.

Desde el punto de vista de uso educativo de la nube, la laaS es adecuada en planes formativos muy diversos y está ampliamente extendida y utilizada en todos los niveles educativos, es la base de lo que se conoce hoy en día como Educación 2.0. Por su parte, el interés sobre las PaaS en la nube proviene fundamentalmente de los estudios relacionados con el desarrollo de software. Por último, los estudios relacionados con los sistemas y redes tienen un nuevo campo de acción con las laaS en la nube, principalmente privados, que permiten manejar y conocer todos los niveles de esta tecnología (Nazareno, G. 2012).

\subsection{Fase de Diseño de Alto Nivel y de Detalle}

En esta fase se diseñó la infraestructura laaS, Figura 3, para mejorar los servicios que provee la ESPAM a sus estudiantes, de forma que las aplicaciones estén en ejecución las 24 horas del día, debido a la robustez de esta infraestructura que se expresa en la estabilidad y alta disponibilidad, y así los servidores virtuales con aprovisionamiento automatizado den constante soporte a las aplicaciones.

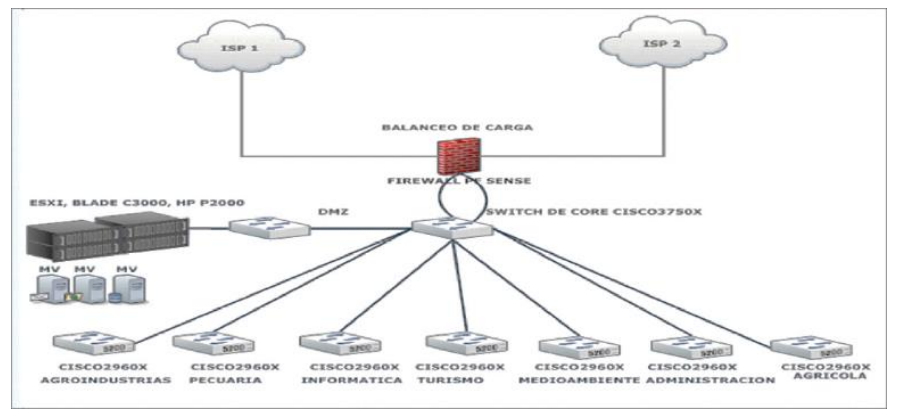

Figura 3. Diagrama de red de laaS del centro de datos de la ESPAM. 


\subsection{Análisis de Redes y Comunicación Redundante}

La Escuela Superior Politécnica Agropecuaria de Manabí no contaba con un centro de datos, principalmente, por ser una institución con pocos años de funcionamiento, adicionalmente los equipos de cómputo que tenía no eran los más idóneos para configurar servidores que garantizaran una continuidad de las aplicaciones provistas a los estudiantes, docentes y personal administrativo.

Actualmente, la universidad cuenta con 1.320 computadoras, de las cuales 463 se destinan a las labores informáticas que realiza el personal administrativo y 857 para las prácticas de laboratorio de los estudiantes. Adicionalmente, la institución dispone de un centro de datos moderno de alta estabilidad, que, con el desarrollo de este trabajo le permite contar con una nube de laaS privada, en la que se han implementado máquinas virtuales, con almacenamiento independiente en arreglos de discos RAID, lo que garantiza que los datos estén preservados mediante mecanismos de respaldo, con la finalidad de salvaguardar la información. Figura 4. Todo este conjunto de acciones le permite a la ESPAM tener un centro de datos de alta confiabilidad de la operatividad de sus aplicaciones y acceso a la información.

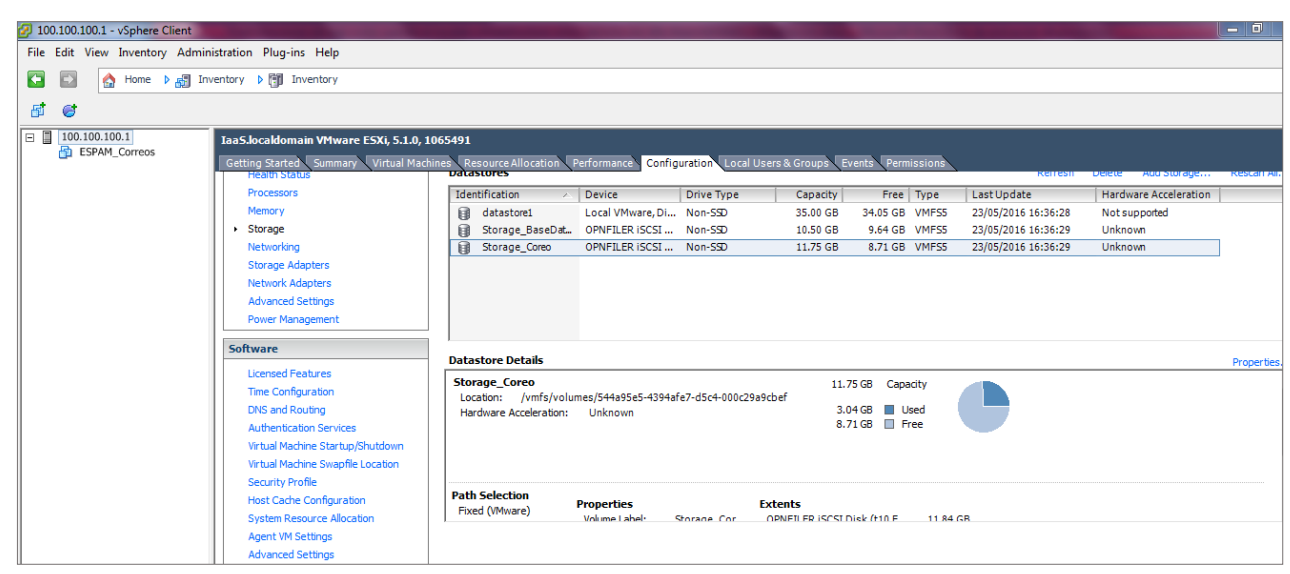

Figura 4. Sincronización con almacenamiento independiente.

Por otra parte, la institución cuenta con un ancho de banda de $60 \mathrm{MB}$, suministrados por TELCONET, dedicados a la comunidad universitaria, y un enlace redundante de $20 \mathrm{MB}$, contratado con CNT, para uso del centro de datos, esto permite realizar un balanceo de carga de los proveedores del servicio de internet.

La seguridad de acceso al centro de datos está controlada por equipos biométricos, dentro del cual los únicos que tienen acceso son el Administrador del Centro de Datos y el Director de la Carreta de Informática. 


\subsection{Fase de Implementación}

Para la ejecución, se realizaron pruebas de sistemas de virtualización libres como propietarios dentro de los cuales se utilizaron OpenNebula, OpenStack, Citrix Xen Server, y VMWare, demostrando este último ser más ergonómico y con más características para poder realizar una administración centralizada haciendo uso del sistema operativo nativo Esxi 5.0 que sería la base para correr las máquinas virtuales, naciendo de esta manera la nube privada de la ESPAM, mismo que contendrá todos los servidores que actualmente se mantienen operativos en la universidad. A raíz de esta implementación, las autoridades, personal docente, personal administrativo y estudiantes en general notaron el cambio tecnológico efectuado en la Escuela Superior Politécnica Agropecuaria de Manabí, dando pauta al crecimiento de la institución. Además, se realizó la integración de las instancias virtuales con el almacenamiento masivo HP P2000, logrando de esta manera una distribución de sistemas estables, confiables, redundantes y de bajo costo.

La segunda fase consistió en la configuración de las máquinas virtuales y la instalación de los sistemas operativos que contendrán las diferentes aplicaciones, como: correo electrónico, entorno virtual de aprendizaje, aplicaciones web, sistemas de matriculación y consulta de notas online, sistema de asistencia de estudiantes, y otros sistemas que se necesiten de acuerdo a la demanda del centro educativo. Figura 5.

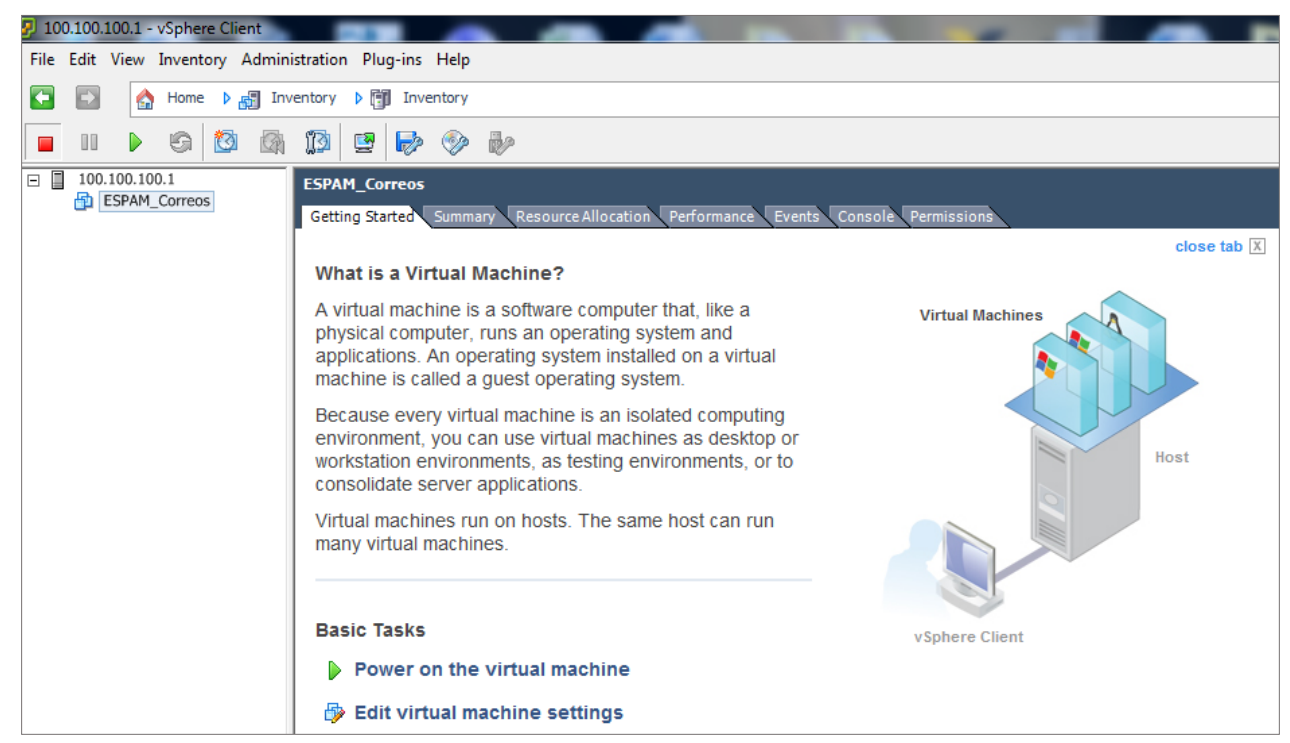

Figura 5. Servidores virtualizados de la ESPAM.

Las ventajas a nivel técnico son fácilmente comprensibles y los beneficios económicos de igual manera. Mostrando mejoras en la efectividad y agilidad organizacional que permite ahorrar costos a las empresas e instituciones educativas. 


\subsection{Fase del Test Unitario, de Integración y Operacional}

Esxi es el sistema operativo o hypervisor más potente de VMware que se instala sobre un servidor físico y crea particiones de múltiples máquinas virtuales, las cuales comparten los mismos recursos físicos que las demás y todas pueden ejecutarse al mismo tiempo sin interrumpirse una de las otras (vmware, 2014). Para las pruebas, realizamos la virtualización de un servidor de correo de manera correcta, pudiéndose visualizar el desempeño en un nivel óptimo, a como se muestra en la Figura 6.

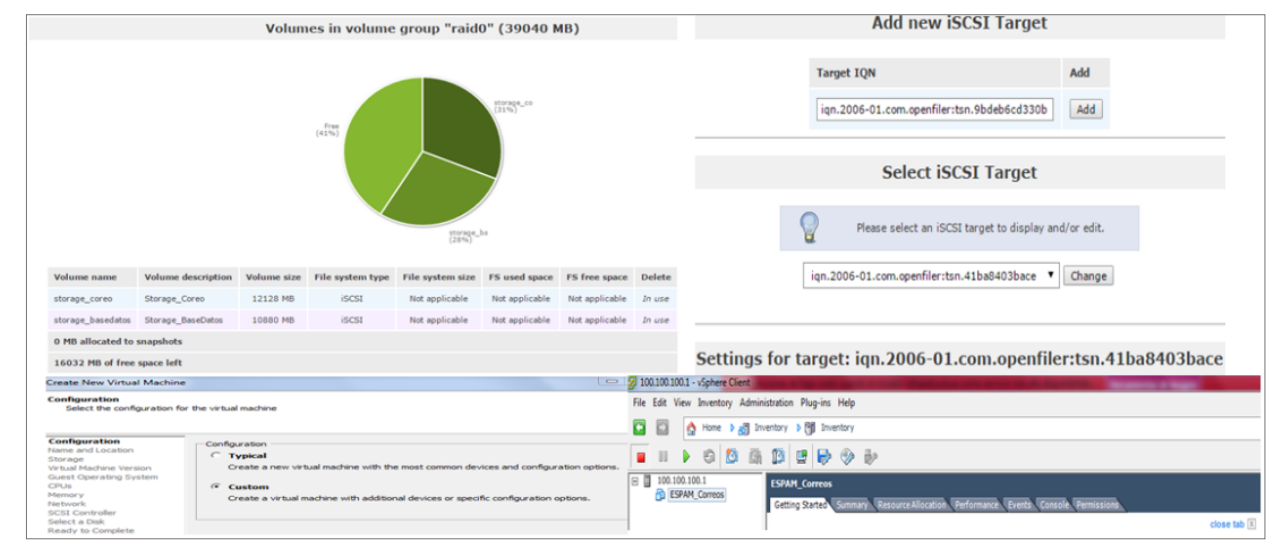

Figura 6. Virtualización del servidor de correos de la ESPAM.

Para realizar los respaldos de cada una de las instancias virtualizadas y de los volúmenes lógicos de almacenamiento se utilizó Nakivo backup replication, lo que permitió minimizar los tiempos de respuestas ante fallos, debido a que es una herramienta que cumple las características apropiadas para realizar los respaldo y restauración en periodos de tiempos cortos, por la tecnología que integra, manteniendo la seguridad en todo momento. Figura 7.

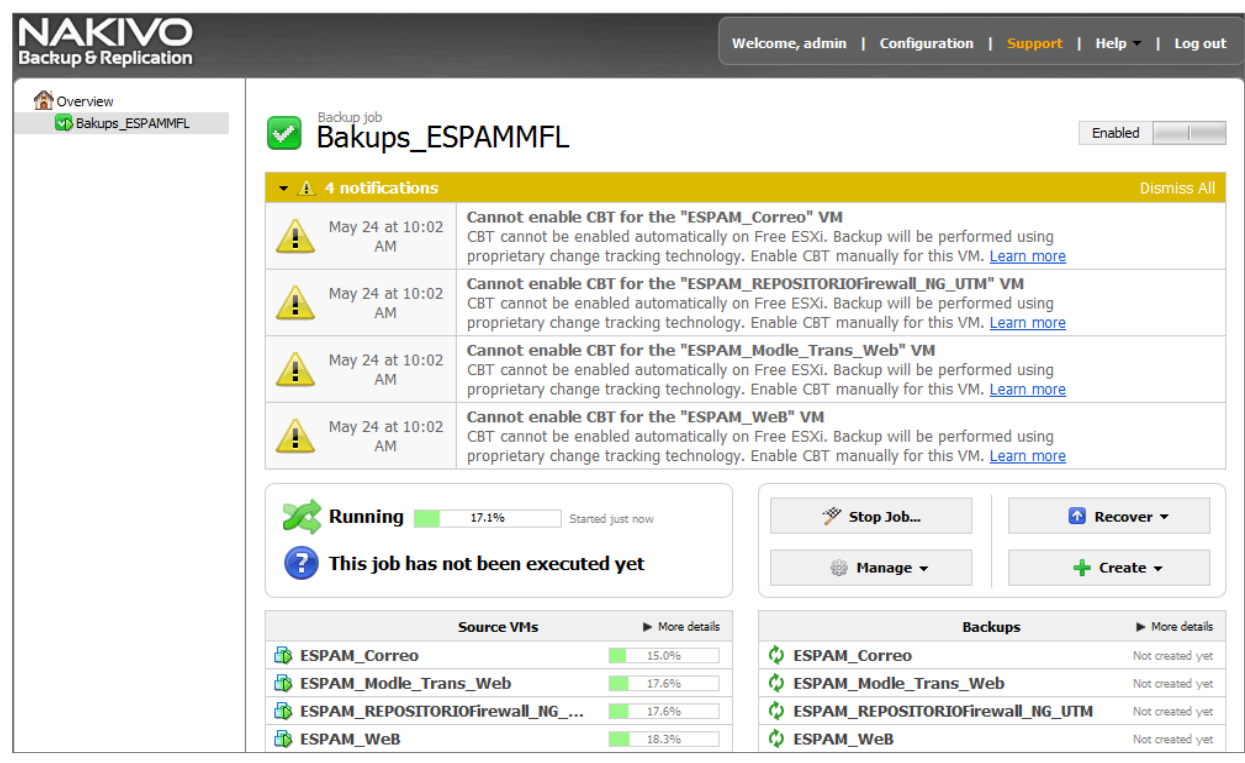

Figura 7. Virtualización de servidor de correo de la ESPAM. 
Otros pruebas realizadas fue la aplicación de vMotion para la migración de máquinas en centros de datos alternos es una tecnología moderna de VMware que permite la migración de las máquinas virtuales desde un host esxi hacia otro host sin perder el servicio, siempre y cuando la configuración de Vmotion se realice adecuadamente tomando en cuenta que para su utilización Vmotion debe estar conectado entre dos host por medio de un medio físico potadora que sea dedicado. Es recomendable la utilización de dos interfaces de red sean estas fiber chanel o iSCSI ya que en vSphere 5 se puede usar múltiples conexiones de red para que el vmkernel balancee la carga entre las dos tarjetas de red logrando con esto la reducción en el tiempo de migración de una máquina virtual desde un host al otro para las pruebas usamos la interfaz vmnic9 como se muestra en la Figura 8.

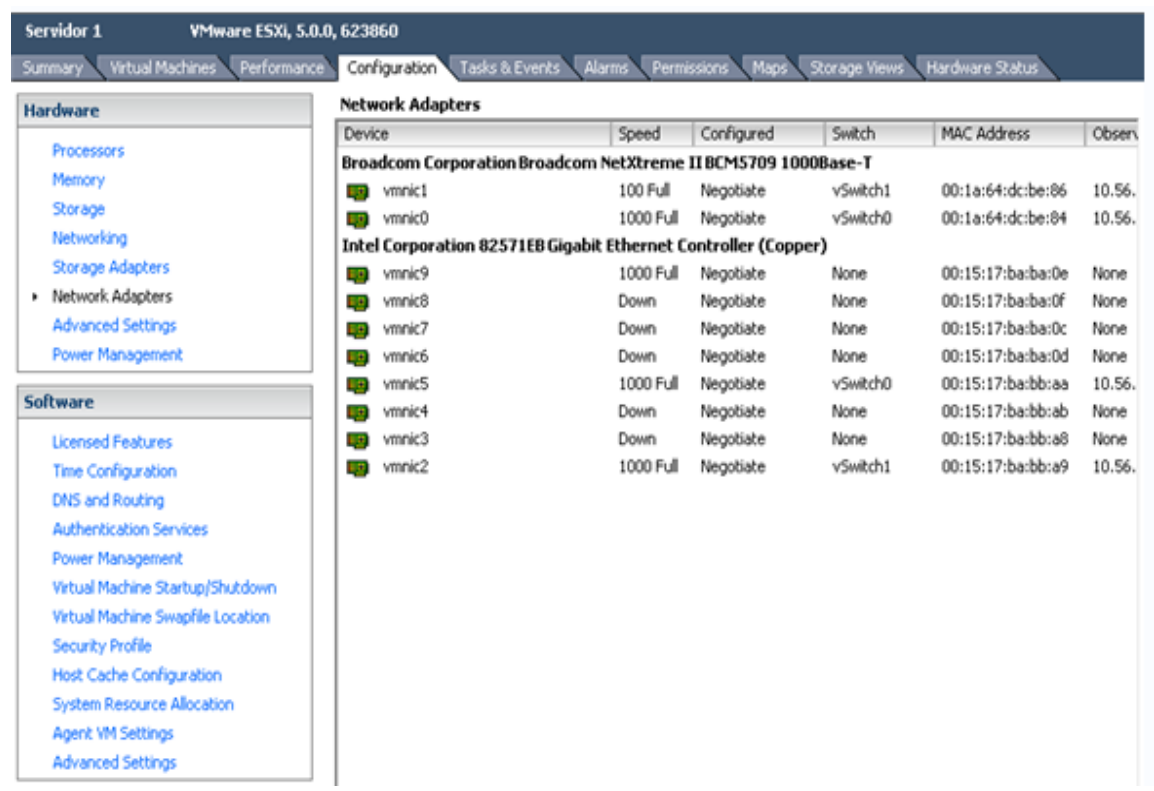

Figura 8. Interfaz vmnic9 que no tiene ningún virtual switch asignado

\section{Resultados y Discusión}

Los resultados que se obtuvieron al implementar en la nube de la laaS del centro de datos de la ESPAM, infraestructura moderna que cuenta con un almacenamiento masivo de 12 discos SAS, de 600 GB cada uno, dos cuchillas incrustadas en el servidor blade C3000 con 2 procesadores Xeon de 64 bits y 16 GB de memoria RAM.

El uso de la virtualización, sistemas redundantes, y la utilización de un sistema RAID conlleva a que se puedan generar volúmenes de almacenamiento masivo en el tiempo de un minuto, mientras que, utilizando la tecnología tradicional, sistemas físicos, se requiere de unos 120 minutos, lo que demuestra la eficiencia de esta técnica, a como se ilustra en la Figura 9. 


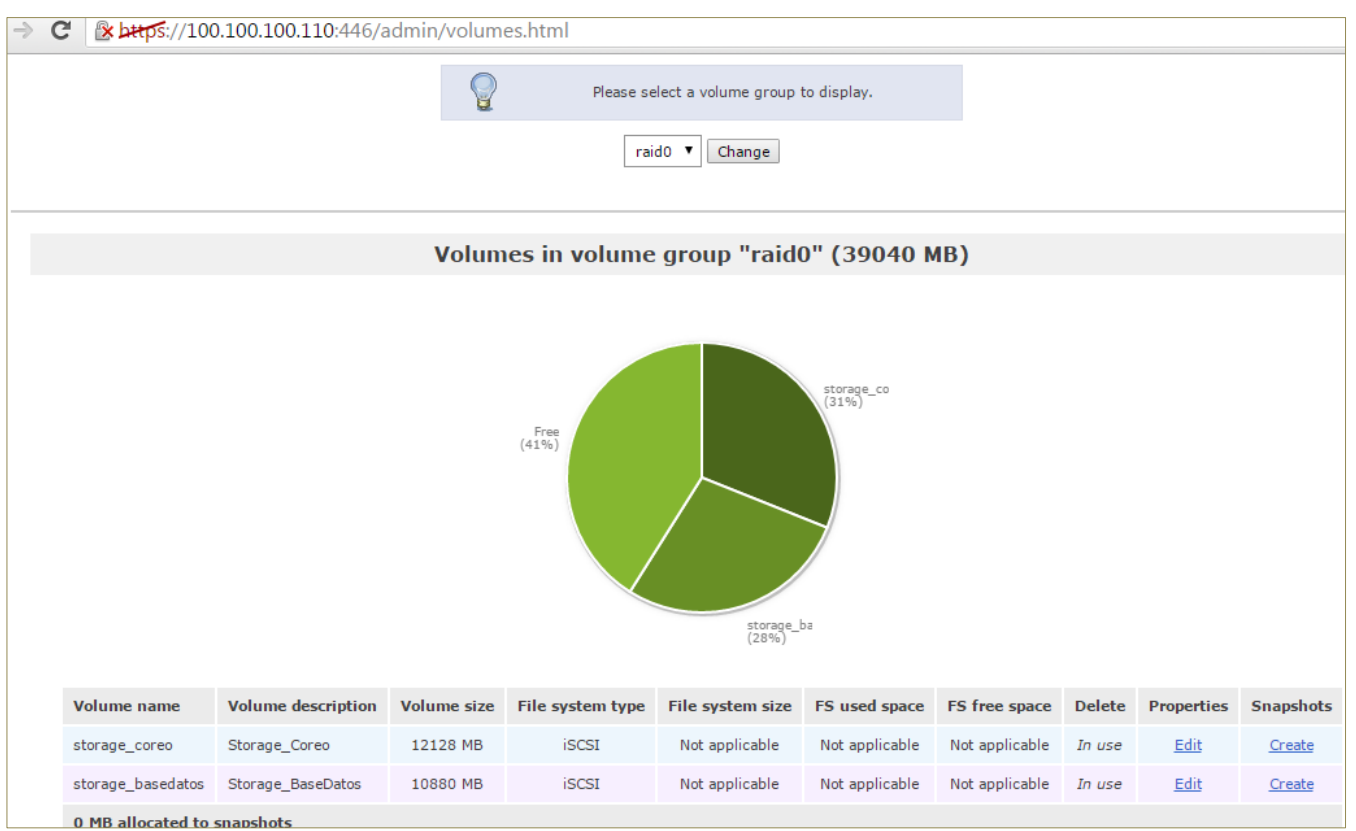

Figura 9. Uso del RAID y volúmenes lógicos de la ESPAM.

Las pruebas realizadas en la infraestructura como servicio en comparación con los servicios tradicionales arrojaron los resultados que se muestran en la Tabla 1.

Tabla 1. Comparativa entre servidores virtuales versus servidores tradicionales.

\begin{tabular}{|l|l|l|}
\hline \multicolumn{1}{|c|}{ Procesos } & \multicolumn{1}{|c|}{ Servidores tradicionales } & \multicolumn{1}{c|}{$\begin{array}{c}\text { Infraestructura } \\
\text { como servicio }\end{array}$} \\
\hline $\begin{array}{l}\text { Adquisición de un nuevo } \\
\text { servidor }\end{array}$ & $\begin{array}{l}5 \text { a } 15 \text { días para la provisión del } \\
\text { nuevo hardware }\end{array}$ & $\begin{array}{l}5 \text { a } 15 \text { minutos para la creación } \\
\text { de una máquina virtual }\end{array}$ \\
\hline Migraciones & $\begin{array}{l}5 \text { a } 8 \text { horas de migración, en } \\
\text { este caso el servicio se } \\
\text { interrumpe. }\end{array}$ & $\begin{array}{l}3 \text { minutos de migración, en } \\
\text { caliente sin interrupción del } \\
\text { servicio. }\end{array}$ \\
\hline Mantenimiento de hardware & 2 a 4 horas de mantenimiento & Casi cero, se hace fuera línea \\
\hline
\end{tabular}

Esta implementación, mediante la virtualización, mejoró la administración, seguridad y disponibilidad de los servicios, aprovechando los recursos del servidor blade C3000, integrándolo fácilmente con un almacenamiento independiente, con lo que se integraron cada uno de los sistemas operativos y las aplicaciones, a como se muestra en la Figura 10. 


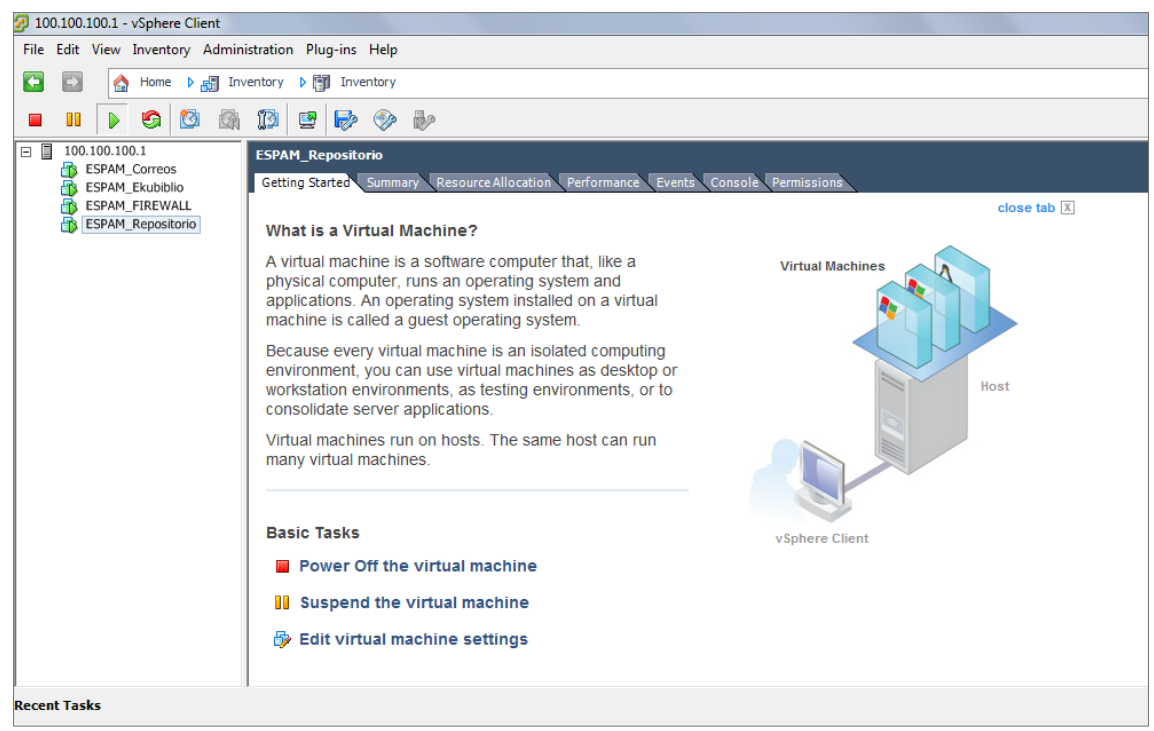

Figura 10. Sistemas operativos y aplicaciones virtualizadas de la ESPAM.

En la Figura 11 se realizan las asignación de IP Cabe indicar que el servidor A debe estar en la misma sub red del servidor B ip servidor A 50.50.50.1, ip servidor B 50.50.50.2 Luego de haber creado la función vMotion en cada uno de los servidores esxi A y esxi B procedemos a realizar la comprobación para la migración de la máquina virtual del host $A$ al host $B$.

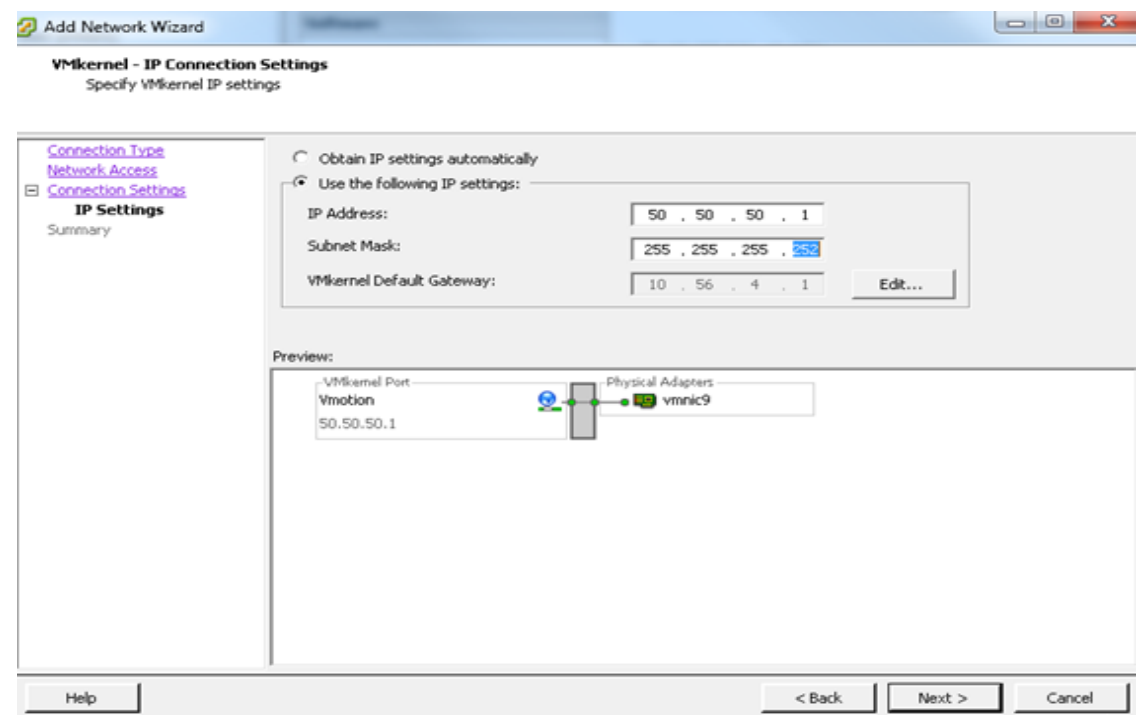

Figura 11. Asignación de la IP correspondientes para tener la conectividad del host A hacia el host B.

El tiempo en realzar la configuración de Vmotion y configuración del vmkernel en cada uno de los host esxi son muy corto alrededor de 5 minutos por host. La migración de la maquinas virtual de un host $A$ hacia un host $B$ haciendo uso de una sola interfaz de red es de para este ejemplo fue de 47 segundo.

La migración de las máquinas virtual de un host $A$ hacia un host $B$ configurado las dos tarjetas de red el tiempo se puede reducir considerablemente a 22 segundos, por que como hemos comentado antes Vmotion es capaz de usar múltiples tarjetas de red para realizar la migración. 
El uso de la laaS en la educación, en la ESPAM, ha permitido ofrecer servicios informáticos en forma de recursos, como mecanismo de ayuda, a los estudiantes en las pruebas, el hardware es configurado a la medida y necesidades de las aplicaciones. Permite, además, una buena agilidad y eficiencia, reduciendo los costes de adquisición e instalación y mejorando la gestión de la información digital de cualquier organización, a través de una implementación sencilla y flexible. Los tiempos que se utilizan para poner en marcha un sistema o aplicación mediante virtualización es de cinco a quince minutos; en cambio, con el sistema tradicional, implementación en hardware, es de una hora y cuarenta cinco minutos.

Al implementar una arquitectura de comunicaciones organizacional con las características de nube e laaS en la Universidad de Cartagena se logra un conjunto de beneficios, todos orientados a la consecución eficiente, sencilla, económica y eficaz de los objetivos de la empresa mediante la optimización de recursos de hardware, software y sobre todo el recurso humano (Tarrá-Reyes, A. et al., 2013).

Los usuarios pueden desplegar máquinas virtuales en la infraestructura física de la laaS en tiempos muy cortos, en los casos críticos en pocos minutos, por lo que se reduce significativamente el tiempo y coste asociado de puesta en marcha de nuevos sistemas. Además, la capacidad de ampliación de los recursos de hardware es bastante menos costosa y rápida que en el tradicional, por lo que se puede incrementar la capacidad de almacenamiento en solo minutos, dependiendo de la necesidad solicitada por el cliente (Orsi, 2010).

El realizar respaldos utilizando Nakivo, permite respaldar y replicar, en centros de datos alternos, todo el sistema operativo en caliente con las diferentes aplicaciones que se encuentren alojadas en el servidor virtual, incluyendo switches virtuales y todas las configuraciones, adicionalmente, lo permite hacer en tiempos muy cortos, unos sesenta minutos; mientras que, utilizando respaldos tradicionales solo se guardan los datos, y para poder guardar también la configuración general del sistema en producción, es decir, respaldar el sistema completo, se requerirá de un mayor tiempo, unas seis horas.

La utilización de sistemas redundantes permite que la información que está en una instancia virtual se replique en otra instancia virtual, manteniendo los mismos datos e información de forma integrada, sean estos hardware de carácter crítico, que se quiere asegurar ante posibles fallos que puedan surgir por su uso continuado, o software, que se pudiere afectar por la manipulación del usuario final o administrador. Lo que se da como una solución a los problemas de protección y confiabilidad en más de una estación, ya que si alguna de las instancias dejara de funcionar o colapsara, inmediatamente otra instancia ocupará su lugar tomando el control.

Migración dinámica de cargas de trabajo vSphere vMotion permite trasladar toda una máquina virtual en funcionamiento de un servidor físico a otro, sin tiempo de inactividad. La máquina virtual 
conserva la identidad y las conexiones de red, lo que garantiza un proceso de migración sin problemas. Transferir la memoria activa y el estado de ejecución preciso de la máquina virtual a través de una red de alta velocidad para que la máquina virtual pase de ejecutarse en el host de origen de vSphere a ejecutarse en el host de destino de vSphere. Todo este proceso dura menos de 22 segundos en una red Gigabit Ethernet. Esta función puede realizarse a través de conmutadores virtuales, vCenter Servers e incluso a grandes distancias.

(Alzoubaidi, A. R. 2016). Para la implementación exitosa de Cloud Computing en las organizaciones debe llevarse a cabo un análisis riguroso para los requisitos del programa y su crecimiento futuro. A la arquitectura de la solución del datacenter, datos, archivo Bases de datos, sistema de almacenamiento, infraestructura y soporte. Servicios necesarios para una solución unificada el cual se realizó en el área salud en nuestra investigación hemos realizado la implementación en educación.

\section{Conclusiones y Recomendaciones}

Mediante la implementación de un sistema de Infraestructura como Servicio, usando la virtualización, en la ESPAM, se mejoró la confiabilidad de los servicios tecnológicos brindados a la comunidad estudiantil. El hecho de ofrecer aplicaciones redundantes, mediante un conjunto de máquinas virtuales que comparten, entre otros, almacenamiento, memoria RAM y procesadores, permite ofrecer una serie de ventajas de capacidad, escalabilidad y disponibilidad en las aplicaciones alojadas en las instancias virtuales, en lugar de tener los servicios ubicados en diferentes servidores dedicados.

Con la implementación del modelo de Infraestructura como Servicio los recursos informáticos de hardware se brindan en forma de servicios. Con ello, la laaS permite aumentar recursos de manera inmediata, en función de las necesidades de los usuarios, así como de las aplicaciones que se encuentren alojadas en el centro de datos; es más, con la virtualización se pueden ampliar o reducir los recursos informáticos en periodos de tiempo muy cortos, de unos cinco minutos.

Los tiempos de recuperación de los servidores, en caso de fallas, son prácticamente inmediatos en comparación con los tiempos de recuperación de los sistemas tradicionales, al beneficiarse de las bondades que ofrece modelo de nube de la laas.

Con las características de servicios que se describen en la Tabla dos, se concluye que la tecnología de Infraestructura como Servicio supera en efectividad a los servidores tradicionales, en cuanto y tanto al cumplimiento de capacidades de integración en servicio de infraestructura y de aplicaciones, porque se ajusta a soluciones estables, con la ventaja de que permite redundancia y replicación en centros de datos alternos, no solo de los servicios y las bases de datos, sino también del sistema operativo completo, además, el fácil aprovisionamiento de almacenamiento hace que los servicios de operación sean factibles. 
Tabla 2. Comparativa de efectividad de servicios entre implementación con laaS versus soluciones tradicionales.

\begin{tabular}{|l|l|c|c|}
\hline Servicio & \multicolumn{1}{|c|}{ Descripción } & Tradicional & laaS \\
\hline Infraestructura & $\begin{array}{l}\text { Redes, almacenamiento redundante distribuido, } \\
\text { sistemas operativos recuperación de } \\
\text { desastres, replicación en caliente, respaldo } \\
\text { integral del sistema con los servicios, } \\
\text { segmentación }\end{array}$ & $15 \%$ & $90 \%$ \\
\hline Aplicaciones & $\begin{array}{l}\text { Seguridad, compartir recursos, interacción, } \\
\text { personalización, disponibilidad, escalabilidad, } \\
\text { servicios y aplicación en línea, replicación en } \\
\text { centros de datos alternos. }\end{array}$ & $5 \%$ & $90 \%$ \\
\hline Operaciones & $\begin{array}{l}\text { Autentificación, disponibilidad, supervisión, } \\
\text { apoyo en la gestión, centro de operaciones de } \\
\text { redes, respaldo. }\end{array}$ & $5 \%$ & $100 \%$ \\
\hline Otros & $\begin{array}{l}\text { Órdenes, aprovisionamiento, licencias, } \\
\text { renovación, mejoras constantes. }\end{array}$ & $1 \%$ & $100 \%$ \\
\hline
\end{tabular}

Con la implementación de Infraestructura como Servicio en la ESPAM, se podrá en un futuro ofrecer recursos máquinas virtuales y almacenamiento como servicios para estudiantes y docentes investigadores, lo que ayudara a expandir más el uso de la tecnología laaS utilizando la infraestructura existente dando servicio a nuevos servidores el clúster virtual.

\section{Bibliografía}

Alzoubaidi, A. R. (2016). Cloud Computing National e-health services: Data Center Solution Architecture. International Journal of Computer Science and Network Security (IJCSNS), 16(9), 1.

Buyya, R.; Yeo, C. S.; Venugopal, S.; Broberg, J.; Brandic, I.: Cloud computing and emerging IT platforms: Vision, hype, and reality for delivering computing as the 5th utility. Future Generation computer systems, Vol. 25, No. 6, pp. 599-616 (2009)

Chuquiguanca, L.; Malla, E.; Ajila, F.; Guamán, R.: Arquitectura Clúster de Alto Rendimiento Utilizando Herramientas de Software Libre. Latin American Journal of Computing Faculty of Systems Engineering National Polytechnic School, Vol. 2, No. 1, pp. $37-44$ (2015).

Guirao Villalonga, A.: Infrastructure as a Service (laaS): application case for TrustedX. http://upcommons.upc.edu/handle/2099.1/11289. (2011). Accedido el 30 de Agosto del 2013.

Nazareno, G.: Cloud Computing en la educacion TIC. http://www.gonzalonazareno.org/cloud/material/cloud_en_la_educacion.pdf. (2012). Accedido el 20 de Noviembre del 2013 
Orsi, Sociedad Informática Castilla.: Cloud Computing la tecnología como servicio. http://www.orsi.jcyl.es/web/jcyl/ORSI/es/Plantilla100Detalle/1262861006271/_/12841523338 22/Redaccion. (2010). Accedido el 27 de Agosto del 2013

Sheharyar, A.; Bouhali, O.: A Framework for Creating a Distributed Rendering Environment on the Compute Clusters. Cornell University Library. http://arxiv.org/abs/1401.0608. (2014). Accedido el 12 de Febrero del 2016

Sociedad española de informática de la salud (2011). Monografico de Cloud Computing. www.seis.es/documentos/noticias/adjunto/seis_monog2011_WEB.pdf. Accedido el 10 de Enero del 2014

vmware. (2014). vSphere ESXi Hypervisor. http://www.vmware.com/co/products/vsphere/features/esxi-hypervisor. Accedido el 08 de noviembre del 2014

Tarrá-Reyes, A. M.; Rodríguez-Ribón, J. C.; Monroy-Ríos, M. E.: Arquitectura para proveer servicios de comunicación soportada en una nube de computación universitaria en el Programa de Ingeniería de Sistemas de la Universidad de Cartagena. Revista Ciencias e Ingeniería al Día, Vol. 8, No. 2, pp. 39-56 (2013)

Zambrano, W.; Chafla, G.; Moreira, C.; Cuzme, F.: Software como servicio de citas médicas en línea, un modelo aplicado a la salud. Espam Ciencia, Vol. 6, No. 1, pp. 31-38 (2015) 